\title{
Secure Mobile Agents Based Clustering Protocol using Modified PSO Algorithm
}

\author{
Nisha A. Auti, Avnish R. Verma, Biplab K. Sarkar
}

\begin{abstract}
As per the studies on Wireless Sensor Network (WSN), the major concern of WSN is network lifespan and Quality of Service (QoS) improvement as the sensor nodes are having limited processing and battery capabilities. The clustering based methods delivered improved performance compared to non-clustering methods, but cluster heads (CHs) suffered from the excessive load which leads more energy consumption of sensor nodes that becomes the $\mathrm{CH}$ in each interval. The mobile agent's technology solves such problems. But using mobile agent's leads problems of data security and unreliable communications in WSN. In this paper, we proposed novel mobile specialists based clustering protocol utilizing the adjusted particle swarm optimization (PSO) algorithm called Secure Mobile Agent based Clustering using modified PSO (SMACP). To optimize the clustering performance we design modified PSO based in $\mathrm{CH}$ selection and cluster formation performed efficiently. To secure data communications using the mobile agents, we applied the trust evaluation scheme to evaluate each mobile agents and sensors using parameters such as residual energy, distance, and packet forwarding probability. The simulation results computed for different WSNs in terms of energy efficiency, average throughput, packet delivery ratio (PDR), also, start to finish delay. The exhibition demonstrates that SMACP outperformed the previous methods effectively.
\end{abstract}

Index Terms: Data aggregation, Clustering, Mobile agents, Security, Particle Swarm Optimization.

\section{INTRODUCTION}

The architecture of sensors nodes shows that the lifespan of a network is principally reliant on the battery of the node [1]. Hence, it is of utmost importance to employ energy efficient protocols for primary tasks i.e. sensing, networking and communication.

In the light of current body of research, sensors have been considered to be deployed in non-deterministic area; hence the deployment, networking and communication shall be carried out efficiently. An in-depth study of literature revealed that lot many system architectures, communication protocols and data aggregation algorithms are available addressing the need of energy efficiency. In a non-deterministic environment, wireless sensors are

\section{Revised Manuscript Received on February 05, 2020.}

* Correspondence Author

Nisha A*. Auti, Maharishi University of Information Technology, Lucknow, India.

Dr. Avanish R. Verma, Maaharishi University of Information Technology, Lucknow, India.

Dr. Bipalab K. Sarkar, TCS, Bavdhan, Pune, India.

(C) The Authors. Published by Blue Eyes Intelligence Engineering and Sciences Publication (BEIESP). This is an open access article under the CC BY-NC-ND license (http://creativecommons.org/licenses/by-nc-nd/4.0/) deployed randomly and they form an ad hoc network [2] Nonetheless, various algorithms supporting the hierarchical clustering for efficient coverage and connectivity and information processing are available in literature and are being discussed in the upcoming sections. However, very few researchers have thought of employing mobile agents to improve the efficiency of these highly useful tiny motes [3]. Although mobile agents have gained a lot of attention in late nineties but very few proposals are available exploiting their capabilities in wireless sensor networks. However, this drifts necessaries sensor hubs to have different capacities to deal with various applications.

Earlier work which used mobile agents in the field of WSN dates back to the year 2005 when the first architecture of Mobile Agent Based Wireless Sensor Network (MAWSN) was given by Chen et al [4]. The system utilizes the mobile specialist's capacity to convey preparing codes that permit the calculation and discussion assets at the sensor hubs to be effectively outfitted in an application particular design. Inferable from their inbuilt features, mobile specialists alter their practices relying upon nature of administration needs (e.g. information conveyance, latency) and the system attributes to expand organize lifetime while as yet meeting those nature of service needs [5]. The mobile agents in WSN offer twin-fold advantages. First of all, mobile agents would move data processing to sensed location resulting into conservation of bandwidth which otherwise would consume lot of energy of sensor nodes, Secondly, mobile agents facilitate collaborative signal and information processing resulting into flexibility of data. Thus interestingly mobile agent based clustering becomes strong research for energy efficiency in WSNs [6].

However, there are number of concerns while using the mobile agents based clustering such as misusing the mobile agents for clustering activities, data accumulation, data combination and so forth in sensor networks, for example, unwavering quality of data transmission, stack adjusting, adaptability, energy effectiveness at both sensor nodes and mobile agents, most imperative is security that may imperilled by mobile agents. From the literature survey, we noticed such difficulties that need to consider while planning the directing conventions for WSNs. In this paper we work on designing the novel secure mobile agents based clustering protocol SMACP to address the challenges of secure communications, efficient clustering and data aggregation for WSNs. In SMACP, first we proposed the modified PSO method to a solution of the issue of unequal energy utilization as well as improving the efficiency while deploying the sensor nodes. After the sensors deployment and clustering using modified PSO, we deployed the mobile agents to gather the information from the Cluster heads (CHs) in network and send it to the sink nodes efficiently. 


\section{Secure Mobile Agents Based Clustering Protocol using Modified PSO Algorithm}

Secondly for the security purpose, trust based algorithm proposed which evaluates the trust score of each mobile agents and sensor nodes before involving in process of communications with $\mathrm{CHs}$ in network using different sensor nodes parameters. In section II, the brief review of different mobile agents based clustering protocols presented.

In segment III, the proposed philosophy of SMACP depicted. In segment IV, the recreation results exhibited. In segment V, conclusion and future scope portrayed.

\section{RELATED WORK}

[7], the creators have proposed another multi-hop mobile information gatherer (MDC) based routing protocol for powerful information conglomeration. Also, the proposed philosophy is better than anything. A Novel Self-Organizing Hybrid Network Protocol for Wireless Sensor Networks (hybrid multi-hop routing protocol) as to node energy usage that overhauls the network lifetime due to multi-hop routing method and keeps up the Start to finish transmission delay.

In [8], another energy protocol called EECMA (Energy Efficient Clustering with Mobile Agents) proposed in view of consolidating the two paradigms of client/server and mobile agent to accomplish the trade-off between increasing the network lifetime and reducing the data conveyance inertness. Moreover, we uncover a multidimensional model to process approaching data from sinks with the end goal to improve the general execution of our system.

In [9] key thought of mobile sinks where mobile sinks are known "data donkeys" proposed. In their work, the mules play out a self-assertive walk around their close-by district to add up to the information bundles and from that point forward, they drop off the information here and there. Since the transmission degree of centres is short, their energy use can be unimaginably lessened.

In [10], the creator proposed a Mobility Based Information Accumulation Calculation recalling the genuine target to improve the network lifetime. In their framework, the zone of the network has a roundabout shape with MSs Working in a forward and in switch advancement in the edges of the circle.

In [11], mobility based strategy with a specific true objective to improve the clustering algorithm and sink hub game plan procedure under the sharp home, the network thought proposed. They thought about two indisputable conditions with a substitute number of mobile sinks. In any case, they utilized a solitary sink that has a circumlocutory improvement in a substitute span. By at that point, they utilized multi mobile sinks to locate the ideal number of mobile sinks in an indirect zone. They thought about that the mobile sinks moved with a relentless speed.

In [12], the speed of the mobile sink is decreased to ensure message transport. This game plan could improve the network lifetime and accomplish a high package movement extent by utilizing diverse mobile sinks with a settled speed.

In [13], author proposed anchored clustered mobile operator based directing convention. Harmony used for securing investigating open enters in a grouped mobile authority WSN to shield sensor center points from malignant directors. Bunch heads go about as a passed on key putting away and question office forming a ring overlay network.

In [14], security mindful mobile specialist based directing convention proposed. They concentrated on making a joined security game plan which uses mobile heads. The proposed security plan utilizing mobile heads is to give central security benefits and to guarantee against seven assaults, for example, Disavowal of Service assault, Hi flooding assault, Traffic examination, Cloning assault, Sink opening assault, Dark hole assault and Explicit sending assault with the energy overhead of watching out for a singular assault.

In [15], the specialist based data collection algorithm for remote networks proposed. They mimicked the operator based predictive data collection algorithm (PLA) and contrasted and ordinary pioneering directing convention.

In [16], author introduced new agenda masterminding algorithm for Mobile Agents (MAs) in light of cluster heads (CHs). As opposed to orchestrating plan among all sensor nodes (SNs), maker organized the calendar just among CHs. In any case, they bundle SNs in bunches in perspective of the thickness of SNs by then select a couple of SNs as CHs. Second, they plan plans for MAs just among CHs in light of minimum spanning over tree algorithm (MST). Finally, maker dispatches a perfect number of MAs for information accumulation and get-together from CHs. In [17], author proposed mobile sink as the mobile specialist for the energy productivity execution change for WSN. They planned the round Movement of Mobile-Sink with Varied Velocity Algorithm (CM2SV2) to modify the energy use degree of cluster heads $(\mathrm{CH})$. Going before playing out the clustering, the creator considered the energy utilization of center points to pick the ideal cluster gauge. A two-sort out Genetic Algorithm (GA) is utilized to pick the ideal between time of cluster measure and get the privilege and motivator from the interim. The proposed SMACP is differing from above works as we first improved the performance of PSO technique before applying it for the mobile agents based clustering. The modified PSO guarantees the lightweight and easy implementation with energy efficiency and energy balance performance. To solve the security related problems, rather than using the complex cryptography based solutions we rely on trust based security method which to establish efficient and reliable communications in network.

\section{METHODOLOGY}

In this paper, the plan of SMACP protocol is presented. Figure 1 illustrates the complete architecture of proposed SMACP protocol. As observed in figure 1, initially the WSNs deployed with random position and mobile agents in network. After the deployment we initiate the tasks of clustering using the modified PSO algorithm. The Cluster Head $(\mathrm{CH})$ selection and cluster formation performed by considering the mobile agents in network. After the clustering process, the next task is data aggregation and dissemination which is performed by different sensor nodes and mobile agents. Mobile agent collects the data from different sensor nodes on behalf of current $\mathrm{CH}$ node in current cluster and disseminates data towards the Base Station (BS) or sink node or other $\mathrm{CH}$ nodes in network. Before performing this, the trust score computed for each sensor node and mobile agents to discover the reliable and secure communication links in network. 


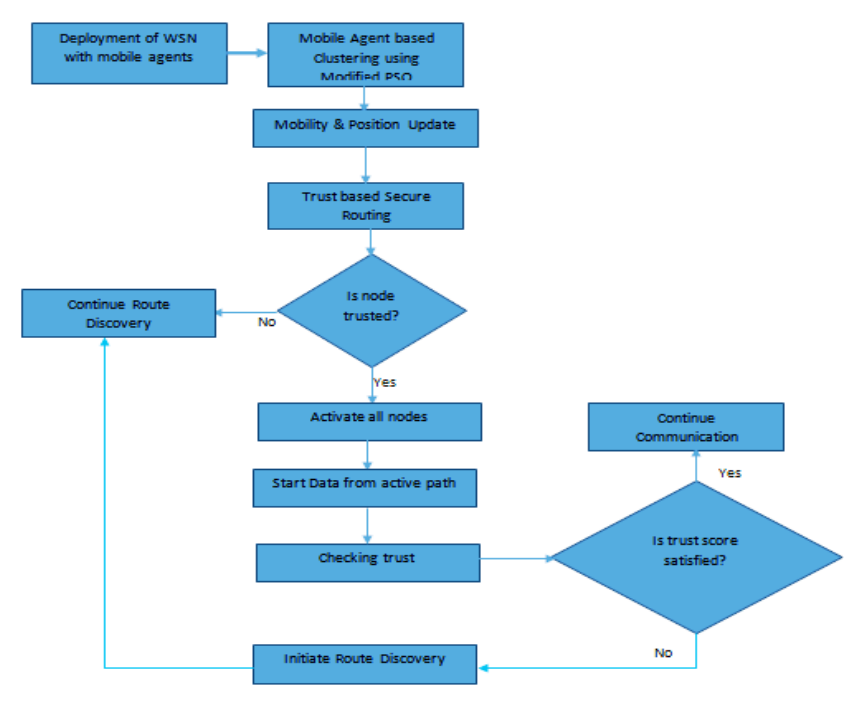

Figure 1: SMACP architecture

If the trust value satisfied, then the mobile agent or sensor node is selected in data transmission path. To save the energy utilization, amid the procedure of course disclosure, the nodes put into the rest mode. Once the route established, those nodes are activated. During the communication, the trust scores periodically checked in order to perform the fault tolerance and improve the reliability of network. Below sections shows the contributions in SMACP.

\section{A. Modified PSO}

The basic PSO algorithm is effectively caught in neighbourhood optima which results in premature convergence. Also, basic PSO is regularly reprimanded is that a few particles may fly far from the hunt region. Therefore, improvement in the PSO algorithm is needed in order to reduce the likelihood of the swarm being trapped into local minima on the error surface and has much better convergence accuracy. Such problems of basic PSO lead challenges of energy balancing for WSNs. To address these problems main the thought is to keep a decent variety of neighbourhood and worldwide optima. A characteristic method to do this is perturbing these optima randomly. In the original PSO perturbation only applies to differences between current position and global best or current position and local best, which result in restricting exploration in search area.

The populaces assume that there is a gathering of $\mathrm{m}$ irregular particles in the d-measurement looking space, the situation of the ith molecule is $x i=(x i l, x i 2, \ldots, x i d)$. The individual best estimation of the molecule is $\mathrm{pi}=$ (pil,pi2,...,pid) and the speed of the molecule is vi = (vil,vi2,...,vid). Therefore, the modified equations (1) and (2) introduced with the aim of widen exploration of particle. By doing this, the perturbations straightforwardly connected to the worldwide best and neighbourhood best:

$v_{i d}=w V_{i d}+\frac{\left[\left(1+r_{3}\right) P_{i d}-X_{i d}\right]}{3}+\left[\left(1+r_{4}\right) P_{g 1}-X_{i d}\right] / 3$

(1)

$X_{i d}=X_{i d}+V_{i d}$

Where $r_{3}$ and $r_{4}$ are uniformly distributed random numbers in [- e, s], where s is a small positive constant. The idea of modified PSO is used in clustering process of WSN using mobile agents in this work.

\section{B. Mobile Agents Based Clustering Using Modified PSO}

Before performing the clustering process, first broadcasting performed after the network deployment. The WSN setup performed in three phases such as broadcasting, clustering, and routing. In broadcasting phase, all the sensor nodes and mobile agents allocates unique ID. The mobile agents collect sensor nodes IDs and other mobile agents in network and then complete local information shared with BS After receiving the information at BS node, the clustering and routing processed initiated. The clustering is performed by using the modified PSO here. The algorithm of clustering is described in below steps:

\section{Algorithm 1: Clustering using Modified PSO}

\section{Inputs}

$\mathrm{N}$ : number of sensor nodes

$\mathrm{d}$ : dimensions of particle $(\mathrm{d}=\mathrm{N})$

$N_{p}$ : predefined size of particles

Pbest: Personal best

Gbest: global vest

$I$ : number of iterations

\section{Output}

Gbest: final clustering solution

1. Particles Initialization: $\mathrm{Let}, \mathrm{Pi}=[\mathrm{Yi}, 1, \mathrm{Yi}, 2, \mathrm{Yi}, 3, \ldots$ , Yi,N] be the ith molecule of the populace where every segment, $Y i, d, 1 \leq i \leq N \_p \quad 1 \leq d \leq N$ maps the task of the sensor node sd to a mobile specialist.

\section{While (I)}

3. Component Initialization:

3.1.Initialize every segment with an arbitrarily created consistently appropriated number Rand $(0,1)$.

3.2. The irregular number is produced autonomously for every part.

3.3. The segment of the dth measurement of this molecule for example Yi,d $=$ rand $(0,1)$ maps mobile operator to which the sensor node sd is doled out. The mapping is done as follows:

\section{4. $m_{k}=\operatorname{Index}\left(\operatorname{ComCH}\left(S_{d}\right), n\right) \quad$ (3)}

where $\operatorname{Index}\left(\operatorname{ComCH}\left(S_{d}\right), n\right)$ is an indexing function that indexes the nth mobile agent from $\operatorname{ComCH}\left(S_{d}\right)$ and $n=\operatorname{celing}\left(Y_{i, d} x\left|\operatorname{ComCH}\left(S_{d}\right)\right|\right)$. The molecule portrayal is a piece of the clustering algorithm. As referenced over that the component of every molecule is equivalent to the quantity of the sensor nodes. In this way, expansion/erasure of any sensor node would change the molecule measurement and require re-clustering.

4. Fitness function derivation: The wellness capacity is determined so that it deals with energy utilization of the $\mathrm{CHs}$ just as the sensor nodes.

The inference relies upon certain parameters portrayed as pursues:

4.1.Lifetime of $\mathrm{CH}$ using Eq. (5)

4.2. Average Cluster distance using Eq. (7)

4.3. Mobility speed using

Eq. (8) 


\section{Secure Mobile Agents Based Clustering Protocol using Modified PSO Algorithm}

\subsection{Fitness value using Eq. (9)}

5. Update Velocity and Position: The speed and the position are refreshed in every emphasis utilizing applying equations (1) and (2)

6. Update Pbest $_{i}$ and Gbest using equations (10) and (11) respectively

7. End While

8. Return Gbest

9. Stop

The algorithm 1 shows the functionality of modified PSO based clustering. The fitness function is based on two important parameters of sensor nods such as network lifetime and average distance.

Lifetime of the CHs: This parameter is chosen to improve the network lifetime while clustering process. This can be conceivable on the off chance that we can boost the lifetime of the mobile operator that has least lifetime. The general rule behind the amplification of the mobile operator life is that the mobile specialist with lower lingering energy ought to have lower rate of energy utilization per round than the mobile specialist with higher leftover energy. In this way the lifetime of the mobile operators with lesser residual energy can be star yearned successfully. The mobile specialists devour their energy for accepting detected information from their part sensor nodes, accumulation of information lastly to send the amassed information to the base station. Along these lines, energy utilization of a mobile specialist g_iwith n_inumber of part sensor nodes due to between cluster movements just as bundle sending is registered as:

$E_{\text {mobileagent }}\left(g_{i}\right)=E_{\text {forwarding }}\left(g_{i}\right) \times E_{\text {clustering }}\left(g_{i}\right)$

The $E_{\text {mobileagent }}\left(g_{i}\right)$ is total energy consumption of mobile agent $g_{i}$. Thus using this, we compute the network lifetime parameter as:

$L\left(g_{i}\right)=\frac{E_{\text {residual }}\left(g_{i}\right)}{E_{\text {mobileagent }}\left(g_{i}\right)}$

Where $E_{\text {residual }}\left(g_{i}\right)$ is residual energy of mobile agent $g_{i}$. The fitness value is proportional of $L$ i.e. $F 1=L$. Thus bigger the estimation of $\mathrm{L}$, higher the wellness esteem.

Average cluster distance: So as to limit the energy utilization of the sensor nodes, they ought to be doled out to their closest $\mathrm{CH}$. Hence, we have estimated the normal separation between sensor nodes and their relating $\mathrm{CH}$.

$A D=\frac{1}{N} \sum_{i=1}^{N} \operatorname{dist}\left(s_{i}, C_{i}\right)$

Where $\mathrm{CH}_{i}$ is the cluster head of sensor node $s_{i}$. The shorter the Promotion, the higher is the wellness esteem. In this manner, the wellness work (F2) is conversely corresponding to the Advertisement, for example

$F 2=\frac{1}{A D}$

Mobility speed: Mobile speed is another factor which can leads to excessive energy consumption in WSNs. The mobility speed helps to control the energy utilization by choosing the mobile agents with less speed. The mobility speed MS of each mobile agents is computed. The shorter the portability speed, the higher is the wellness esteem. Hence, the wellness work (F3) is contrarily corresponding to the Advertisement, for example

$F 3=\frac{1}{M S}$

By considering all above parameters the fitness value computed as:

Fitness $=F 1+F 2+F 3$

After the wellness esteem calculation, we update the speed and position. In the event that new position is more prominent than one, at that point supplant the position an incentive by one. Subsequent to getting the new position, the molecule $\mathrm{Pi}$ is assessed by the wellness capacity utilizing Eq. (9). Presently, its own best (Pbesti) is supplanted independent from anyone else, just if its present wellness esteem is superior to anything its Pbesti wellness Esteem:

Pbest $=\left\{\begin{array}{cc}P_{i} \text { if }(\text { fitness }(P i)<\text { fitness }(\text { pbesti })) \\ \text { Pbesti }\end{array}\right.$

Presently, the worldwide best is additionally refreshed as pursue:

Gbest $=\left\{\begin{array}{cc}P_{i} \text { if }(\text { fitness }(P i)<\text { fitness }(\text { Gbesti })) \\ \text { Gbesti }\end{array}\right.$

The speed and the positions are iteratively refreshed until the end criteria are satisfied. In this work, the end foundation is a predefined cycle number. After end of the adjusted PSO-based routing algorithm, the molecule Gbest speaks to the last clustering arrangement.

\section{Trust based Routing}

After the clustering process, the process of data aggregation and dissemination performed by computing the trust score of mobile agents or sensor nodes in network. We compute the trust score of each node that involve in process of sending data from source sensor node to BS using the parameters like network lifetime, average distance, and packet delivery probability. The parameters network lifetime and average distance are computed for each node similar ways as described above.

\begin{tabular}{|c|c|c|}
\hline \\
\hline \multicolumn{3}{|l|}{$\begin{array}{l}\text { Algorithm 2: Secure Communication } \\
\text { Inputs } \\
\text { S: Source Node } \\
B S: \text { Sink }\end{array}$} \\
\hline & $\begin{array}{l}1 . \\
2 . \\
3 . \\
4 . \\
5 . \\
\\
\\
6 . \\
7 . \\
\\
8 . \\
9 . \\
10 . \\
11 . \\
12 . \\
13 . \\
14 . \\
15 .\end{array}$ & $\begin{array}{l}S \text { initiates the route discovery } \\
S \text { finds one-hop neighbouring nodes } n \\
S \text { broadcast RREQ's to } n \\
\text { Put all } n \text { in sleep mode } \\
\text { Upon receiving RREQ at each I } \epsilon n \\
\text { 5.1. Network lifetime computing using Eq. (5) } \\
\text { 5.2. Average distance computation using Eq. (7) } \\
\text { 5.3. Packet delivery probability using Eq. (12)-(14) } \\
\text { Compute the trust using eq. (15) } \\
\text { Select intermediate node } I \text { with maximum value } T^{I} \\
\text { among all } n \\
\text { Update routing table } \\
\text { If (I == D) } \\
\quad \text { Active all nodes in path } \\
\quad I \text { sends RREP to } S \\
\quad S \text { starts data transmission } \\
\text { Else } \\
\text { Repeat } S=I \\
\text { End If }\end{array}$ \\
\hline
\end{tabular}

For computing the packet delivery probability, we estimate the available and demanded bandwidth of particular node. This can be as: 


$$
\begin{aligned}
& A_{b}+R_{b}<\sigma, T=1 \\
& A_{b}+R_{b}=\sigma, T=0 \\
& A_{b}+R_{b}>\sigma, T=-1
\end{aligned}
$$

Where $A_{b}$ bandwidth allocated at current node and $R_{b}$ is required bandwidth to transmit the current data, and $\sigma$ is lower bandwidth limit to satisfy. The value of $T$ is used in trust computation process further.

Trust $=(F 1+F 2+T) / 3$

\section{SIMULATION RESULTS}

To investigate the performance of SMACP we designed WSNs using the NS2 simulator with varying number of sensor nodes, for example, 200 to 700 with aggregate of 60 mobile specialists to perform the tasks of data collection and aggregation to achieve the load balancing and energy efficiency in network. The SMACP performance is compared with MAPA [16] and CM2SV2 [17]. The network parameters presented in table 1 used to configure the WSNs.

\begin{tabular}{|c|c|}
\hline Number of sensor nodes & $200-700$ \\
\hline Simulation Time & 100 second \\
\hline Mobile Agents (MAs) & 30 \\
\hline Mobility of MAs & $10 \mathrm{~m} / \mathrm{s}$ \\
\hline Routing Protocols & $\begin{array}{lll}\text { MAPA, CM2SV2, and } \\
\text { SMACP }\end{array}$ \\
\hline MAC & IEEE 802.11 \\
\hline Propagation Model & Two-Ray Ground \\
\hline Mobility & $\begin{array}{l}\text { random waypoint mobility } \\
\text { model }\end{array}$ \\
\hline Antenna & Omni Antenna \\
\hline CBR Connections & 5 \\
\hline Network size & $500 \times 500$ \\
\hline Packet size & 512 \\
\hline Initial energy (SNs \& MNs) & $30 \mathrm{~nJ} / \mathrm{bit}$ \\
\hline Mobile Agents & 60 \\
\hline
\end{tabular}

Table 1: Reproduction parameters Number of sensor nodes

The performance measured as far as normal energy utilization, network lifetime, and normal throughput to guarantee the throughput to claim the QoS of networks and energy efficiency. Figure 2 (table 2) and 3 (table 3) demonstrates the outcomes of energy efficiency performances of SMACP with both recent clustering

Retrieval Number: E7405068519/2020@BEIESP

DOI: 10.35940/ijeat.E7405.029320

Journal Website: www.ijeat.org methods investigated. The results demonstrates that as the quantity of sensor nodes builds, the energy consumption in network increases as well and hence leads lower network lifetime. The performance of CM2SV2 method shows the better energy efficiency performance as compared to MAPE technique. The CM2SV2 method mainly designed to tackle the energy imbalanced issue in which the mobile sinks are used as MAs to overcome the problem of energy hole. However, the key problem with this method is there is manual required to adjust the speed of mobile sinks in various zones so as to adjust the energy utilization of Cluster heads (CHs). The problems with CM2SV2 and MAPE protocols addressed by proposed SMACP which improves the performance of energy efficiency compared to both protocols as the efficient PSO algorithm used for clustering and trust ware data communications.

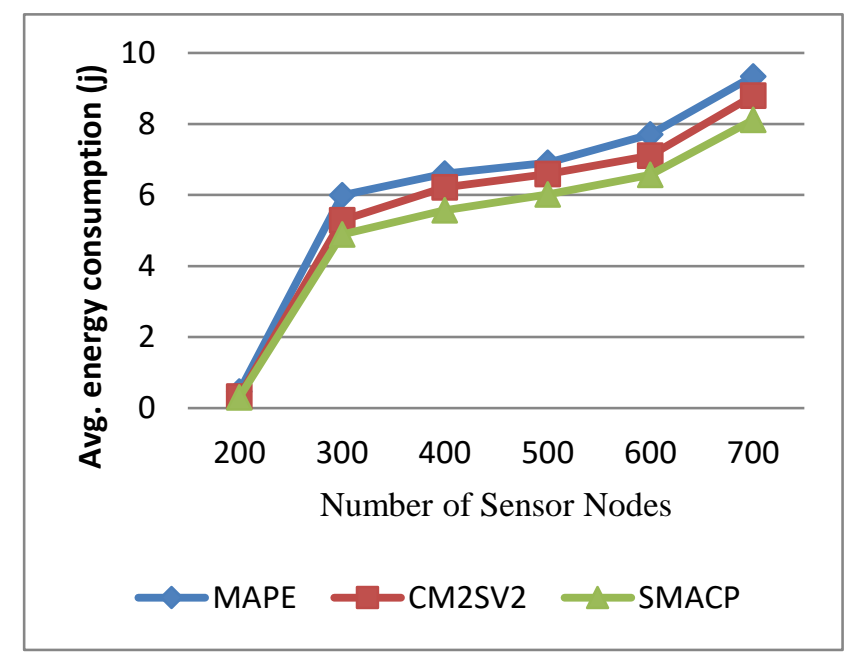

Figure 2: Average energy consumption analysis

Table 2: Readings of average energy consumption

\begin{tabular}{|c|c|c|c|}
\hline & MAPE & CM2SV2 & SMACP \\
\hline 200 & 0.467 & 0.32 & 0.3 \\
\hline 300 & 5.99 & 5.29 & 4.89 \\
\hline 400 & 6.6 & 6.22 & 5.57 \\
\hline 500 & 6.91 & 6.59 & 6.01 \\
\hline 600 & 7.71 & 7.11 & 6.57 \\
\hline 700 & 9.34 & 8.79 & 8.11 \\
\hline
\end{tabular}

Figure 4 demonstrated the average throughput performance analysis. The throughput performance of MAPE algorithm shows the better improvement as compared CM2SV2 algorithm, as the MAPE designed for efficient data aggregation using the MAs in clustering. The outcomes of both existing methods are contradictory; it means they failed to achieve the trade-offs between the energy and QoS efficiency for the sensor networks. 
However the SMACP achieves the trade-off between QoS and energy efficiency performances as compared to CM2SV2 and MAPE protocols.

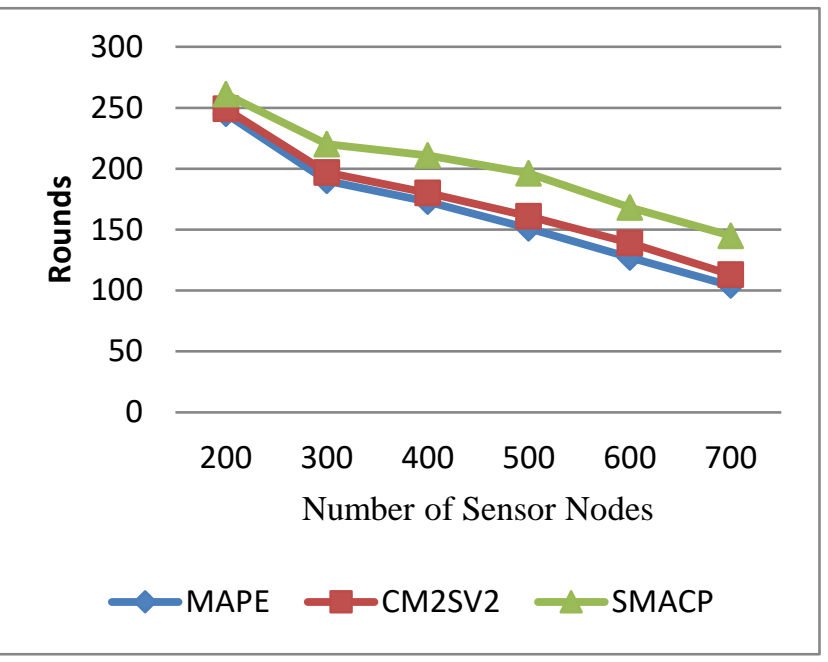

Figure 3: Network lifetime performance analysis

Table 3: Readings of network lifetime performance

\begin{tabular}{|c|c|c|c|}
\hline & MAPE & CM2SV2 & SMACP \\
\hline 200 & 245 & 249 & 261 \\
\hline 300 & 190 & 197 & 220 \\
\hline 400 & 173 & 180 & 211 \\
\hline 500 & 151 & 161 & 196 \\
\hline 600 & 127 & 139 & 168 \\
\hline 700 & 104 & 113 & 145 \\
\hline
\end{tabular}

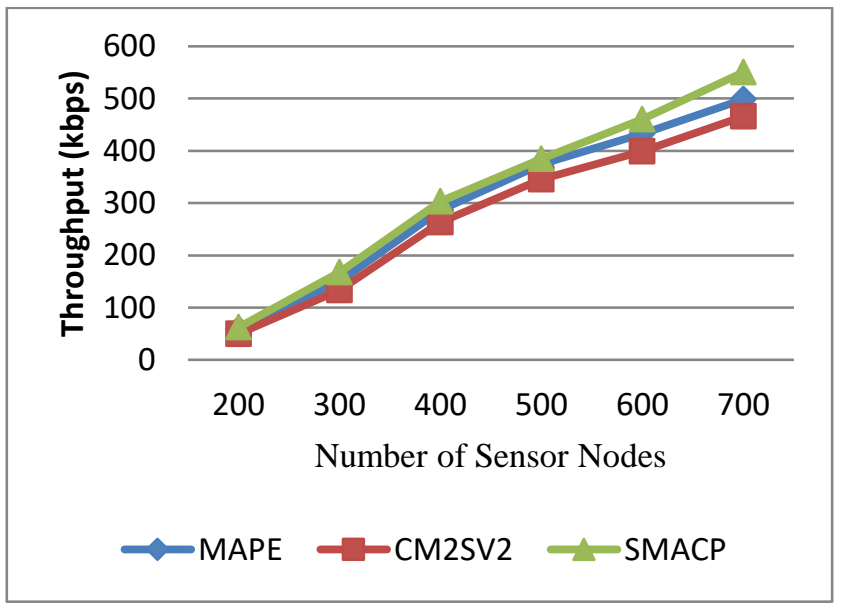

Figure 4: Average throughput performance analysis

Table 4: Readings of average throughput performance

\begin{tabular}{|c|c|c|c|}
\hline & MAPE & CM2SV2 & SMACP \\
\hline 200 & 58.81 & 49.84 & 62.3 \\
\hline 300 & 149.89 & 133.45 & 167.9 \\
\hline
\end{tabular}

\begin{tabular}{|c|c|c|c|}
\hline 400 & 286.82 & 263.74 & 303.4 \\
\hline 500 & 374.56 & 345.33 & 384.5 \\
\hline 600 & 432.34 & 398.78 & 460.4 \\
\hline 700 & 498.55 & 465.89 & 550.4 \\
\hline
\end{tabular}

The performance of SMACP improved compared to recent methods due to two key points (1) the use of modified PSO algorithm for mobile agents based clustering which achieved the better convergence accuracy and energy balance in network effectively (2) use of trust aware and modified PSO based data aggregation and dissemination algorithm which heals the unreliable communications caused by either mobile agents or sensor nodes in network.

\section{CONCLUSION AND FUTURE WORK}

In this paper to comprehend the challenges related to optimized mobile agents clustering and security, we proposed the mobile agent based clustering by designing novel SMACP protocol which not only heals the network security but also improves the performance in terms of energy efficiency, average throughput, average delay, and PDR performances. The SMACP is based on two key contributions such as mobile agents clustering using modified PSO and trust aware data aggregation and dissemination. The experimental studies conducted using NS2 tool for different types of networks. The performance of SMACP is compared with recent mobile agent based protocols for large WSNs. The results claim that SMACP achieved the trade-off between the network QoS and energy efficiency. For future work we suggest: (1) Design the real time application of WSNs using SMACP ex. Military, security applications, monitoring etc.

(2) Design and investigation of SMACP for IoT enabled WSNs, and (3) Evaluations of different network attacks.

\section{REFERENCES}

1. Cagalj, M., Hubaux, J.-P., and Enz, C. C., "Energy-efficient broadcasting in all-wireless networks," Wireless Networks, 11(1/2), 177-188, 2005.

2. Polastre, J., Szewczyk, R., and Culler, D., "Telos: Enabling ultra-low power wireless research," In Proceedings of international symposium on information processing in sensor networks (pp. 364-369), 2005.

3. Chen, Y. P., Wang, D. and Zhang, J., "Variable-base tacit-communication: a new energy efficient communication scheme for sensor networks," Proceedings of the First International Conference in Integrated Internet Ad Hoc and Sensor Networks, InterSense 2006, Nice, France, May 30-31, 2006.

4. Chen, Y. P., Liestman, A. L., \& Liu, J., "Energy-efficient data aggregation hierarchy for wireless sensor networks," In Proceedings of 2nd international conference on quality of service in heterogeneous wired/wireless networks (QShine '05), Orlando, 2005

5. Bharat Sundararaman, Ugo Buy and Ajay D. Kshemkalyani, "Clock Synchronization for Wireless Sensor Networks: A Survey", Journal of Ad Hoc Networks, Vol.3,2005, pp. 281-323.

6. Chuan Zhu, Chunlin Zheng, Lei Shu, and Guangjie Han, "A Survey on Coverage and Connectivity Issues in Wireless Sensor Networks", Journal of Network and Computer Applications Vol. 35, No.2,2012, pp. 619-632.

7. Muhammad Arshad, Mohamad YahyaAalsalem, Farhan Ahmed Siddqui, "Multi-hop routing protocol for Mobile Wireless Sensor Networks", DOI: 10.1109/WCCIT.2013.6618712. 
8. Rais Amine, Kada Amine, Bouragba Khalid, and Ziyati El houssaine, "Knowledge discovery in WSN using mobile agents", DOI: 10.1109/ISACV.2015.7106161,2015.

9. Shah, R.C.; Roy, S.; Jain, S.; Brunette,W, "Data MULEs: Modeling a three-tier rchitecture for sparse sensor networks", Ad Hoc Netw. 2003, 1, 215-233.

10. Wang, J.; Zuo, L.; Shen, J.; Li, B.; Lee, S., "Multiple mobile sink-based routing algorithm for data dissemination in wireless sensor networks", Concurr. Comput. 2015, 27, 2656-2667.

11. Wang, J.; Yin, Y.; Zhang, J.; Lee, S.; Sherratt, R.S., "Mobility based energy efficient and multi-sink algorithms for consumer home networks", IEEE Trans. Consum. Electron. 2013, 59, 77-84.

12. Ma, J.; Chen, C.; Salomaa, J.P. " $\mathrm{mWSN}$ for large scale mobile sensing”, J. Signal Process. Syst. 2008, 51, 195-206.

13. Alyaa Amer Ayman, Abdel-Hamid Mohamad, Abou El-Nasr, "Chord-Enabled Key Storage and Lookup Scheme for Mobile Agent-Based Hierarchical WSN", Advances in Security of Information and Communication Networks. SecNet 2013. Communications in Computer and Information Science, vol 381. Springer, Berlin, Heidelberg.

14. D. Sheela, G. Mahadevan, "Mobile Agent Based Enhanced Security for Wireless Sensor Networks", International Journal of Business Data Communications and Networking archive Volume 10 Issue 2, April 2014

15. Rupali Rohankar, "Agent Based Predictive Data Collection in Opportunistic Wireless Sensor Network", Procedia Computer Science, Volume 57, 2015, Pages 33-40.

16. Mohamed El Fissaoui, Abderrahim Beni-Hssane, Mostafa Saadi, "Mobile Agent Protocol based energy aware data Aggregation for wireless sensor networks", Procedia Computer Science Volume 113, 2017, Pages 25-32.

17. Niayesh Gharaei, Kamalrulnizam Abu Bakar, Siti Zaiton Mohd Hashim, "An Energy-Efficient Mobile Sink-Based Unequal Clustering Mechanism for WSNs”, Sensors (Basel). 2017 Aug; 17(8): 1858.

\section{AUTHORS PROFILE}

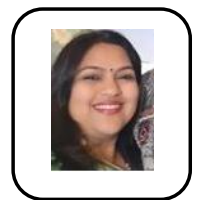

Nisha A. Auti, has completed BE, ME in computer Engineering and perusing Ph.D in computer Engineering from Maharshi University Lucknow.. He has total 15 years of teaching Experience and working as a Asst. Professor at TSSM Pune She has published 5 Scopus indexed journal papers and 2 patents.

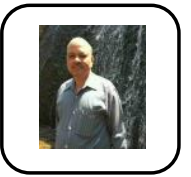

Dr. Avnish Sharma has completed BE, ME and Ph.D in computer Engineering and working as a Professor in Computer department for Maharshi University Lucknow.. He has vast experience in teaching.

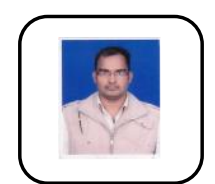

Prof. Dr. Biplab Kumar Sarkar, Post.Doc. (Singapore) PhD.(CS)(IIT- BHU), M-Tech(CS) IIT-BHU, B-Tech(CS). Member. IETE, ISTE, SMU, IIHT, Global $\mathrm{R} / \mathrm{D}, \mathrm{GEH}$. He is having 19 years of academic as well as industrial experience. He holds various positions like He has worked on many funded project. Since 2003 total approx.1200 Cr. Governmental/ Non-Governmental project completed. He is having 185 patent filled and 03 is under provisional patent process. He is associated with 05 various universities. He has published 10 books in Engineering field and 02 is under process . He has published 20 papers in national/International journals and conference. 